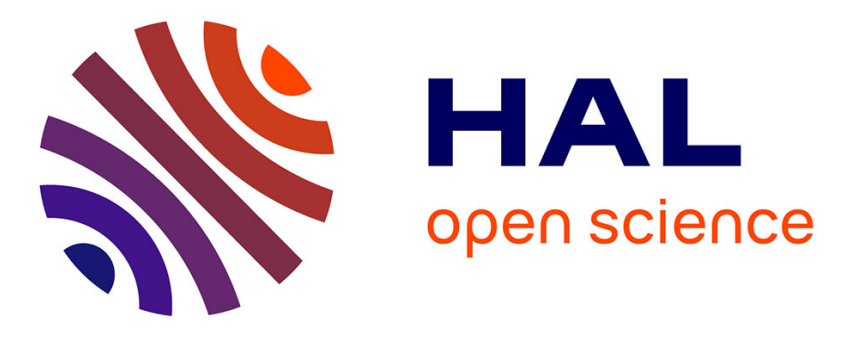

\title{
Model-based control of slugging: advances and challenges
}

Florent Di Meglio, Glenn Ole Kaasa, Nicolas Petit, Vidar Alstad

\section{To cite this version:}

Florent Di Meglio, Glenn Ole Kaasa, Nicolas Petit, Vidar Alstad. Model-based control of slugging: advances and challenges. IFAC Workshop on Automatic Control in Offshore Oil and Gas Production 2012, May 2012, Trondheim, Norway. hal-00706862

\section{HAL Id: hal-00706862}

https://hal-mines-paristech.archives-ouvertes.fr/hal-00706862

Submitted on 11 Jun 2012

HAL is a multi-disciplinary open access archive for the deposit and dissemination of scientific research documents, whether they are published or not. The documents may come from teaching and research institutions in France or abroad, or from public or private research centers.
L'archive ouverte pluridisciplinaire HAL, est destinée au dépôt et à la diffusion de documents scientifiques de niveau recherche, publiés ou non, émanant des établissements d'enseignement et de recherche français ou étrangers, des laboratoires publics ou privés. 


\title{
Model-based control of slugging: advances and challenges
}

\author{
F. Di Meglio* G.-O. Kaasa ${ }^{* *}$ N. Petit ${ }^{* * *} \operatorname{Vidar}^{*}$ Alstad $^{* * * *}$ \\ * University of California San Diego, 9500 Gilman Drive, La Jolla, CA, \\ 92093 USA (e-mail: fdimeglio@ucsd.edu). \\ ** Statoil ASA, Research Center Porsgrunn, Heroya Forskningspark 3908 \\ Porsgrunn, Norway (e-mail: gkaa@statoil.com). \\ *** Centre Automatique et systèmes, MINES ParisTech, 60 bd St-Michel, \\ 75272 Paris Cedex 06, France (e-mail: nicolas.petit@mines-paristech.fr). \\ **** Yara Technology Center, Heroya Forskningspark 3908 Porsgrunn, \\ Norway (e-mail: vidar.alstad@yara.com).
}

\begin{abstract}
We review recent advances in the suppression of the slugging phenomenon by model-based control. We focus on three aspects of recent contributions: models, observers and control laws. For each category, we evaluate and compare existing solutions, and propose directions for improvement.
\end{abstract}

\section{INTRODUCTION}

We investigate recent advances in the field of model-based control of the slugging phenomenon. We focus on the contributions in terms of modelling, estimators and feedback control laws.

Slugging is an undesirable multiphase flow phenomenon arising on oil production facilities, mainly wells and flowline risers. The main concern associated with slugging is that it causes the level of production to decrease. The mechanisms of these losses, corresponding to a bifurcation behavior first described in Zakarian [2000], are schematically summarized by Figure 1, where the level of production is plotted against the opening of the outlet valve for a slugging well. For high values of the opening, the production periodically oscillates in time. The average of these oscillations is lower than the equilibrium production, which is a first cause for production losses. More importantly, these oscillations may damage the pipe, and hurt the separation process. For this reason, operators are forced to close the outlet valve, which stabilizes the flow (as evidenced by the pioneer works of Schmidt et al. [1979]), but yields a lower level of production. This is the second and main cause for production losses, which motivates the need for control laws that enable one to operate at open-loop unstable operating points.

Among the viable solutions to cope with the slugging phenomenon, we focus in this paper on feedback actuation of the outlet valve ${ }^{1}$, as first proposed in Blick et al. [1986]. Successful implementations of PI controllers using pressure sensors in feedback loops have been reported since the 1990s Courbot [1996], Hedne and Linga [1990]. In order to improve the performances of controllers, one can rely on dynamical models reproducing the behavior of slugging systems. After describing existing models, we review how they are utilized to design observers and control laws well-suited to the slugging problem.

The article is organized as follows. In Section 2, we review the existing dynamical models reproducing the slugging behavior. In Section 3, we describe how these models, along with ap-

\footnotetext{
1 other solutions include reducing the diameter of the pipe or installing slug catchers, and are usually less cost-efficient.
}

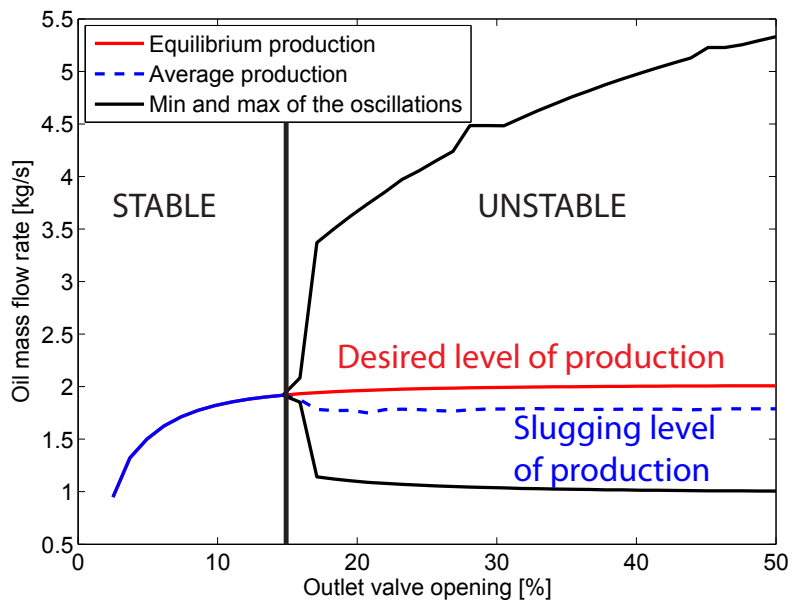

Fig. 1. Schematic view of the impact of slugging on the level of production.

propriate measurements, can be used in observer algorithm to estimate missing information. In Section 4, we detail different control strategies from the literature, before giving practical conclusions in Section 5.

\section{MODELLING OF THE SLUGGING PHENOMENON}

In this section, we review and compare existing models reproducing the two-phase slugging phenomenon, dividing them into two categories: distributed parameters models and finite dimensional models.

\subsection{Distributed parameter models}

The systems subject to the slugging phenomenon, oil wells and flowline risers, consist of pipes ranging from several hundreds to several thousands meter-long pipes filled with oil, gas, and sometimes water. The distributed nature of these very long systems naturally suggests models consisting of Partial Differential Equations (PDE). Whereas these complicated models are traditionnaly used for simulation purposes only, recent contributions 
propose to directly derive observers Bloemen et al. [2006] or control laws Di Meglio et al. [2012] from PDE models.

Simulation-oriented models PDE models for two-phase flow are derived by writing mass and momentum conservation laws for the gas and liquid phases on infinitesimal section of the pipe. They can, again, be divided into two classes of models described in greater details in Duret [2005].

- Two-fluid models feature separate momentum equations for the gas and the liquid. The most notable two-fluid model is the one embedded in the commercial multiphase flow simulator OLGA ${ }^{\mathrm{TM}}$ Bendiksen et al. [1991], which serves as a reference. It is able to reproduce the slugging phenomenon observed on most systems, and is especially accurate at steady-state.

- Conversely, drift-flux models feature a combined momentum (and sometimes energy) equation for the gas and the liquid, while an empirical slip equation relates the velocities of the two phases. Numerical implementations showing the relevance of such models can be found in Belfroid et al. [2010], Henriot et al. [2002].

Regardless of the class they belong to, none of the PDE models described in the literature have been, to our best knowledge, directly used for control or observer design purposes. Existing results rely on a discretization in space of these infinite-dimensional models in view of a controllability analysis Storkaas [2005] or the design of an Extended Kalman Filter (EKF) Bloemen et al. [2006].

In the next section, we briefly describe a simplified model, which takes into account the distributed nature of the phenomenon while remaining simple enough for control design.

A PDE model for control In Di Meglio et al. [2011], we describe a drift-flux model for two-phase slugging flow, able to reproduce the slugging phenomenon. The model consists of two mass conservation laws, written for the gas and liquid phases, respectively, and one combined momentum equation. The main simplifying modelling assumption lies in the slip relation, which is assumed, following Sinègre [2006] to take the following form

$$
v_{g}-v_{l}=\frac{v_{\infty}}{\alpha_{l}}
$$

where $v_{g}$ is the gas velocity, $v_{l}$ the liquid velocity, $\alpha_{l}$ the liquid hold-up. $v_{\infty}$ is here a constant tuning parameter whereas, on most drift-flux models, it is assumed to depend on the physical states of the model, following various possible empirical relations (see e.g. Brennen [2005]). This allows us to write the system equations in closed form as a three-state quasilinear hyperbolic system as follows

$$
\frac{\partial \mathbf{u}}{\partial t}(t, x)+A(\mathbf{u}) \frac{\partial \mathbf{u}}{\partial x}(t, x)=G(x)
$$

where $\mathbf{u}(t, x)$ is the vector of states comprising, as components, the mass fraction of gas, the pressure and the velocity of gas, defined on the space domain $x \in[0, L], L$ being the total length of the pipe. Along with appropriate boundary conditions, this model reproduces the slugging phenomenon, as illustrated by the simulations depicted on Figure 2, where the evolution of the liquid mass fraction and gas velocity profiles is presented.

2.1.2.1. Linear control design After linearization around an equilibrium profile, the model takes the form of the following
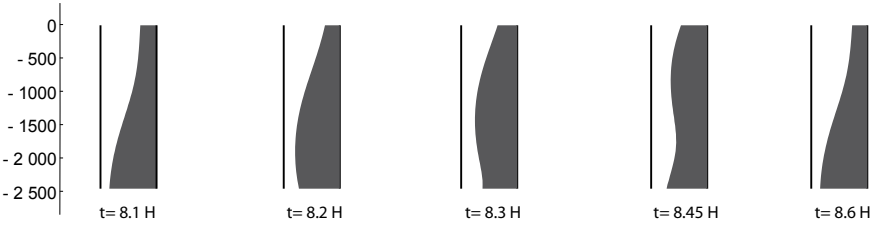

(a) Liquid mass hold-up profiles
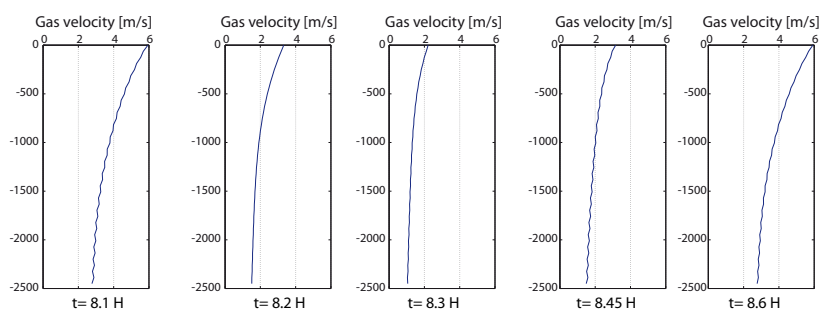

(b) Gas velocity profiles

Fig. 2. Liquid mass hold-up and gas velocity profiles at different time instants. At $t=8.1 \mathrm{~h}$, a slug is formed at the bottom of the pipe. It is then transported upwards and expelled over the next .5 hours, while another slug is formed. The velocity of the flow is inhomogeneous in space and time: the slugging cycle comprises phases of acceleration and deceleration, as pictured on Figure (b).

$3 \times 3$ linear hyperbolic system of transport equations with spacedependent velocities and source term coefficients

$$
\begin{aligned}
& u_{1_{t}}(t, x)+\lambda_{1}(x) u_{1 x}(t, x)=0 \\
& u_{2 t}(t, x)+\lambda_{2}(x) u_{2 x}(t, x)+\sigma_{21}(x) u_{1}(t, x)+\sigma_{23}(x) v(t, x)=0 \\
& v_{t}(t, x)-\mu(x) v_{x}(t, x)+\sigma_{31}(x) u_{1}(t, x)+\sigma_{32}(x) u_{2}(t, x)=0
\end{aligned}
$$

where the states $u_{1}, u_{2}$ and $v$ represent small variations around an equilibrium profile, but have lost part of their physical interpretation through state transformations ${ }^{2}$. The boundary conditions for this system are expressed as follows

$$
\left(\begin{array}{l}
u_{1}(t, 0) \\
u_{2}(t, 0)
\end{array}\right)=\left(\begin{array}{l}
q_{1} \\
q_{2}
\end{array}\right) v(t, 0) \quad v(t, 1)=U(t)
$$

where $q_{1}$ and $q_{2}$ are non-zero coefficients, and $U(t)$ is the control input, related to the opening of the outlet valve. The fact that $U$ appears only in the right boundary condition in Equation (5) suggests the use of boundary control techniques for PDE. In Di Meglio et al. [2012], a full-state boundary feedback law yielding exponential stability of the $\left(u_{1}, u_{2}, v\right) \equiv(0,0,0)$ equilibrium of system (2)-(4) is designed, using a backstepping approach. In details, the destabilizing coupling terms $\sigma_{i j}$, $i, j=1,2,3$ are canceled using a backstepping transformation transforming system (2)-(4) into an exponentially stable target system. For more details on boundary control design using backstepping, the interested reader is referred to Krstic and Smyshlyaev [2008a,b], Vazquez et al. [2011].

To our best knowledge, the control law derived in Di Meglio et al. [2012] is the first to directly handle destabilizing terms arising from the distributed nature of the phenomenon. This should prove efficient in stabilizing expansion-driven slugging

\footnotetext{
2 More precisely, $u_{1}$ represents small variations of the gas mass fraction around the equilibrium profile. The two other states, $u_{2}$ and $v$, may be interpreted as pressure waves traveling in opposite directions inside the pipe, but are related to the original physical state variables in a non-trivial way which is hard to interpret.
} 
or the density-wave phenomenon. However, the proposed controller is not implementable as is, since it requires full information on the distributed states of the system, which is not realistic in practice. For this reason, the design of a boundary observer using topside measurement is currently under investigation, and should be the subject of future contributions.

This analysis, although promising, stresses the need for simpler, finite dimensional, models, for which control theory provides a vast array of tools in view of stabilization. We now review such existing models for slugging.

\subsection{Finite dimensional models}

In this section, we briefly describe four lumped parameters models: the Jansen model for gas-lifted wells, the Storkaas and Jahanshahi models for risers with low-points, and the Di Meglio model, for vertically inclined wells. This list is not exhaustive and other efforts include the models proposed in Kaasa et al. [2007], Da Silva and Nydal [2010]. An extensive comparison of the capabilities of these different models is presented in Jahanshahi and Skogestad [2011].

To neglect the distributed nature of the slugging phenomenon, finite dimensional models rely on strong simplifying modelling assumptions. After reviewing the mechanisms generating instability in these models, we focus on two counterparts of such simplifications: the calibration effort and the limited representativeness of these models.

\section{Mechanisms and model structure}

\subsubsection{Mechanisms The first finite-dimensional models} have been designed for very specific classes of systems, for which the causes of the slugging instability are well understood. Such models include the Jansen model studied in Eikrem et al. [2002], Imsland [2002], Jansen et al. [1999], Sinègre [2006] for gas-lifted systems, and the Storkaas and Jahanshahi models described in Jahanshahi and Skogestad [2011], Storkaas and Skogestad [2002] for flowline risers with low-point. For both these classes of systems, instability is generated by a blockage of gas at a certain location in the system. In the case of gaslifted wells, the gas contained in the casing can only enter the tubing if the pressure drop over the injection valve is positive. In the case of risers with low point, the liquid, accumulating at the low-point angle, acts as a valve blocking the flow of gas there. In both cases, the blockage of gas causes a buildup of pressure, followed by a "blow-out" where gas and liquid abruptly accelerate and exit the pipe at high flow rates, before the slugging cycle repeats.

This mechanism has been extended to a broader class of systems in Di Meglio et al. [2009, 2010a]. The main assumption of the Di Meglio model is the existence of a irregularity, causing the blockage of gas, and modeled as a "virtual valve". On the two classes of systems mentioned above, the irregularity is clearly identified as the gas injection valve for gas-lifted wells, and the low-point angle in the case of flowline risers. By extrapolating this mechanism to, e.g., vertically inclined wells, we are able to reproduce the slugging behavior of some systems for which no finite dimensional models existed. This is the case of the well schematically depicted on Figure 3(a), for which we have plotted the measured and reconstructed pressure variations on Figure 3.

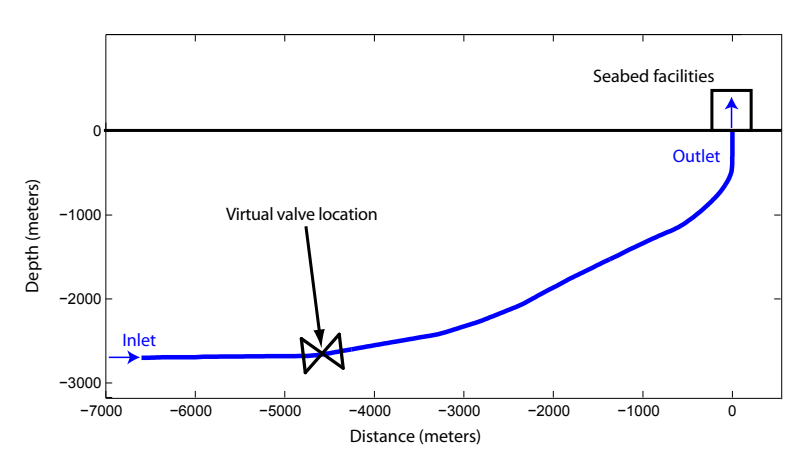

(a) Schematic view of a 5700 meter-long well in the North Sea. The well does not feature any low-point angle, nor is it gas-lifted.
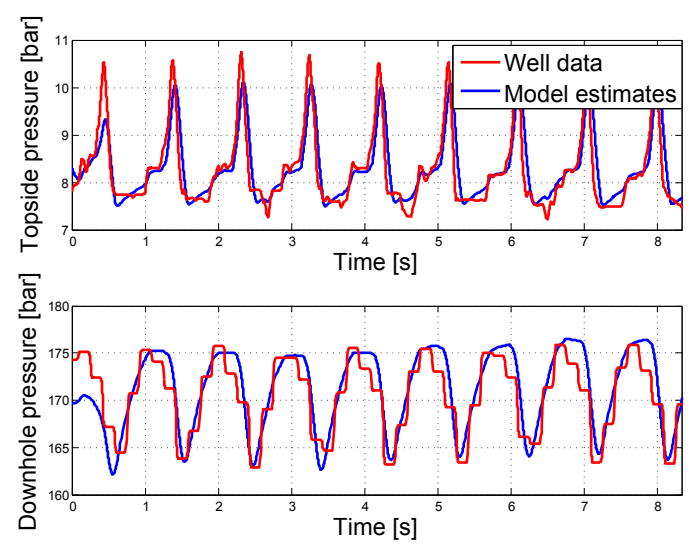

(b) Measured and reproduced pressure oscillations for the well depicted on Figure 3(a).

Fig. 3. Geometry and pressure oscillations of a 5700 meter-long well in the North Sea.

2.2.1.2. Structure of the models These models, excepting the one of Jahanshahi Jahanshahi and Skogestad [2011], share a common structure that arises from the mechanisms described above. The state variables are two masses of gas $\left(m_{1}\right.$ and $m_{2}$, here) separated by a valve, and one mass of liquid $\left(m_{3}\right)$. The mass conservation laws for these three masses read

$$
\begin{aligned}
& \frac{d m_{1}}{d t}=(1-\epsilon) w_{g, \text { in }}(t)-w\left(m_{1}, m_{2}, m_{3}\right) \\
& \frac{d m_{2}}{d t}=\epsilon w_{g, \text { in }}(t)+w\left(m_{1}, m_{2}, m_{3}\right)-w_{g, \text { out }}\left(m_{1}, m_{2}, m_{3}\right) \\
& \frac{d m_{3}}{d t}=w_{l, \text { in }}\left(m_{1}, m_{2}, m_{3}\right)-w_{l, \text { out }}\left(m_{1}, m_{2}, m_{3}\right)
\end{aligned}
$$

where $w_{g, \text { in }}$ (resp. $w_{l, i n}$ ) is the total mass flow rate of gas (resp. liquid) into the system, and $w_{l, \text { in }}$ (resp. $w_{l}$,out) the total mass flow rate of liquid into (resp. out of) the system. $w$ represents the mass of gas through the injection or virtual valve and $\epsilon$ is a design parameter. In the Jahanshahi model, an additional state is considered, corresponding to the mass of liquid upstream the low-point. Similarly to the flow of gas, the flow of liquid through the low-point is assumed to satisfy a valve equation.

The detailed equations can be found in Imsland [2002] for the Jansen model, in Storkaas [2005] for the Storkaas model and in Di Meglio [2011] for the Di Meglio model. They comprise several parameters which may require some tuning, as detailed in the next section.

Calibration effort The simplification of considering finite dimensional models has an important counterpart in the cal- 
ibration effort. Indeed, some parameters of the models may lose some of their physical meaning as empirical relations between physical states and modelling assumptions are considered. When it is the case, tuning procedures must be defined to find the "right" values for these parameters, in a sense that we now define.

Calibration is needed to ensure that the models reproduce quantitatively the behavior of a given system. The goal of a tuning procedure is therefore to fit the dynamical properties of the model to that of the system. A large part of these properties is summarized by the bifurcation diagram of the bottom pressure. For this reason, the tuning procedures proposed in Storkaas [2005] for the Storkaas model and in Di Meglio et al. [2010b] for the Di Meglio model both match certain parameters with a characteristic of the bifurcation diagram. For example, in Di Meglio et al. [2010b], the volume of gas located upstream the virtual valve can be calibrated to fit the position of the bifurcation point.

Although most of the values of the parameters can be found analytically, as the mathematical tractability of the models increase, part of the tuning procedures remains on a trial-anderror basis. This is one of the limitations of finite dimensional models that we review in the next section.

Limitations and challenges As a rule, there seems to be a trade-off between the simplicity of the model, which allows for a greater mathematical tractability (in particular, the tuning procedure becomes more analytical as the models get simpler), and representativeness of the model. We now give an example that highlight this feature, comparing the model for gas-lift proposed in Imsland [2002], Sinègre [2006], and our model first described in Di Meglio et al. [2009].

In the model for gas-lifted wells described in Imsland [2002], the inflow of oil coming from the reservoir is assumed to depend linearly on the pressure difference between the reservoir and the pipe. The so-called Productivity Index relating these two quantities is usually known with an acceptable accuracy. This relation allows the model to highlight the crucial mechanism through which slugging causes production losses: the average pressure during slugging oscillations is higher than the equilibrium production, which causes the level of production during slugging to be lower than the equilibrium level.

However, in the model described in Di Meglio et al. [2009], the inflow of liquid is assumed constant. The Productivity Index relation is not needed to generate instability in the model, and a constant liquid inflow greatly simplifies the analysis. It allows, e.g., to write the equilibrium of the model in closed-form, which would not be possible with a pressure-driven inflow. A constant inflow is one of the reason why such a large part of the tuning procedure proposed for our model in Di Meglio et al. [2010b] is analytical, which is of course desirable.

This example shows that a challenge for future models should be to find the right balance between physical meaningfulness and mathematical tractability. Besides, despite the generalization provided by the model presented in Di Meglio et al. [2009], numerous systems feature behaviors that could not be accounted for using existing finite dimensional models. Existing models cannot, e.g., reproduce the behavior of a well featuring several slugs present at the same time in the pipe. For other occurrences of slugging, such as the density-wave phenomenon Hu [2004], Sinègre [2006] or expansion-driven

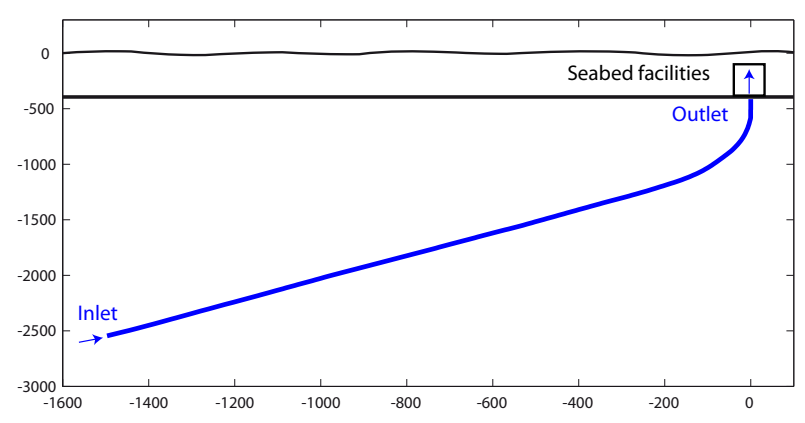

(a) Schematic view of a 2700 meter-long well in the North Sea. The well does not feature any low-point angle, nor is it gas-lifted.
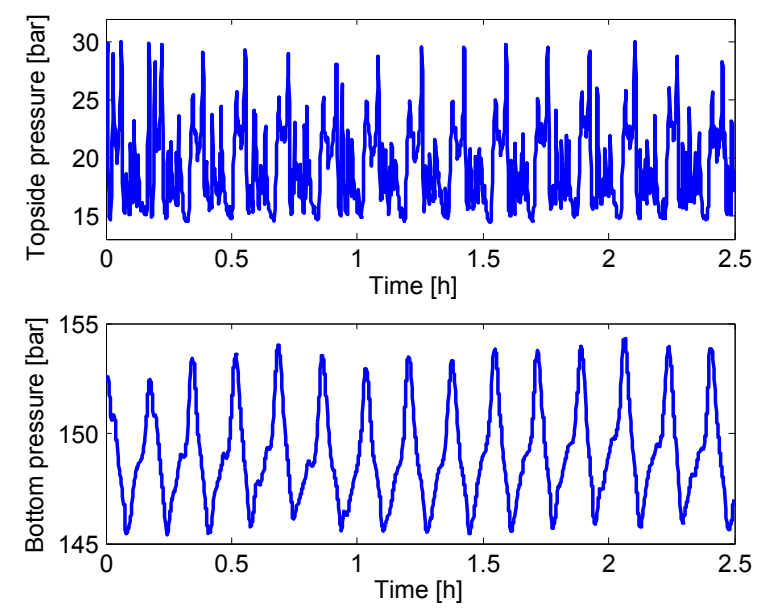

(b) Measured oscillations for the well depicted on Figure 4(a). The shape and frequency of the oscillations differ significantly from those of the well of Figure 3, and could not be reproduced with the model described in Di Meglio et al. [2009]

Fig. 4. Geometry and pressure oscillations of a 2700 meter-long well in the North Sea.

slugging, the distributed nature of the phenomenon cannot be neglected, and a PDE model is required.

To illustrate these limitations, Figure 4(b) pictures the pressure oscillations observed on a slugging well, that we will refer to as the A-well, schematically depicted on Figure 4(a). The frequency of the oscillations is much higher than these pictured on Figure 3(b), in proportions that cannot be explained by the shorter length of the A-well alone. In turn, the slugging seems to involve here different mechanisms than the one modeled by the "virtual valve", that are yet to be understood. None of the finite dimensional models discussed above were able to reproduce the behavior of the A-well.

Despite these shortcomings, when the models are representative of the considered systems, they can be used to estimate missing measurements from available sensors, as described in the next section.

\section{OBSERVERS}

In this section, we review various observer algorithms from the literature. Various underlying models and combination of sensors are considered. The majority of the observers described in the literature are designed for gas-lift systems. This is of great importance since the measurements available topside (i.e., in 
easily accessible locations) are in greater number than for wells without gas-lift. We first review several estimation strategies for gas-lifted wells, before describing an observer design for simple vertical wells.

\subsection{Observers for gas-lifted wells}

Extended Kalman Filters (EKF) Several contributions consider the implementation of an EKF (see, e.g. Kailath et al. [2000]) relying on nonlinear models for slugging. The EKF consists in applying the linear Kalman Filter to the linearized dynamics around the current estimation.

- In Bloemen et al. [2006], a discretized drift-flux model (see Section 2.1) is considered. The EKF is first used considering a distributed array of pressure sensors, which is not realistic in practice. A second scenario considers topside sensors only (both in the casing and the tubing), and highlights the necessity of a liquid flow rate measurement for the estimation of certain state variables ${ }^{3}$.

- In Eikrem et al. [2004], an EKF is used to estimate the states of the finite dimensional model for gas-lifted wells discussed in Section 2.2. The proposed scheme relies on measurements of the topside and bottom pressures, and presented simulations show that the availability of the bottom pressure sensor is critical. The algorithm is tested in simulations, where the estimates, despite featuring a relatively small steady-state error, are used to stabilize a gas-lifted well modeled in OLGA ${ }^{\mathrm{TM}}$.

Nonlinear observers A different class of observers for slugging directly relies on the nonlinear models, rather than their linear approximation. Most of these designs rely on the Jansen model Jansen et al. [1999], Imsland [2002] for gas-lifted wells discussed in Section 2.2. We now review several contributions, corresponding to various combinations of available sensors and different estimation strategies.

- In Sinègre [2006], an estimator for the bottom pressure, using topside information from the casing only (both pressure and inflow rate of gas), is presented. To decouple the casing and tubing dynamics, the bottom tubing pressure is assumed to vary slower than the bottom casing pressure. This simplification yields an easily implementable observer, but no proof of convergence of the scheme is provided. The design is validated both in simulations and on real-well data, but a control scheme using the measurement in a closed-loop feedback law is only tested on simulations.

- In Aamo et al. [2005], a nonlinear observer relying on topside measurements from both the casing and the tubing is designed for the Jansen model. The three measured outputs are the topside casing pressure, topside tubing pressure and topside tubing outflow rate ${ }^{4}$. A Lyapunov analysis is used to prove convergence of the scheme, which provides estimates for the three state variables of model (6)-(8). Presented control experiments on midscale experimental facilities illustrate the relevance of the approach by depicting stabilization of a slugging system using the estimated bottom tubing pressure as the controlled variable.

\footnotetext{
3 in particular, the gas bubbles rise velocity

4 the flow measurement can be replaced by a density measurement.
}

- Similarly, in Scibilia et al. [2008], a high-gain observer relying on topside measurements from both the casing and the tubing is designed for the Jansen model. The observer features linear output injection functions, which is the main difference with the observer design from Aamo et al. [2005]. This simplifies the implementation of the observer, but no proof of convergence of the complete scheme is provided. The estimates are used to stabilize a slugging system in simulations, with success.

\subsection{An observer for vertical wells}

The design of observers for simple vertically inclined wells is more challenging than for gas-lifted wells, because of the number and location of available measurements. In particular, the measurements from the casing of gas-lifted well constitute "upstream" information which is not available in the case of simple wells. In this section, we describe an observer design for the Di Meglio model first proposed in Di Meglio et al. [2010b].

3.2.0.1. Observer design The aim of this observer design is to provide estimates for the states of model (6)-(8) using a measurement of the topside pressure only. The observer equations are obtained by, rewriting system (6)-(8) with the new set of variables $\left(m_{1}, y, m_{3}\right)$, where the measured output $y$ is the topside pressure. The observer then consists of a copy of the system plus a linear output injection term, as described by the following equations

$$
\begin{gathered}
\text { Model equations } \\
\left\{\begin{array} { l l } 
{ \dot { m } _ { 1 } = f _ { 1 } ( m _ { 1 } , y , m _ { 3 } ) } \\
{ \dot { y } } & { = f _ { 2 } ( m _ { 1 } , y , m _ { 3 } ) } \\
{ \dot { m } _ { 3 } } & { = f _ { 3 } ( m _ { 1 } , y , m _ { 3 } ) }
\end{array} \quad \left\{\begin{array}{ll}
\dot{\hat{m}}_{1}=f_{1}\left(\hat{m}_{1}, \hat{y}, \hat{m}_{3}\right) \\
\dot{\hat{y}}=f_{2}\left(\hat{m}_{1}, \hat{y}, \hat{m}_{3}\right)-k(\hat{y}-y) \\
\dot{\hat{m}}_{3}=f_{3}\left(\hat{m}_{1}, \hat{y}, \hat{m}_{3}\right)
\end{array}\right.\right.
\end{gathered}
$$

3.2.0.2. Qualitative analysis As of now, we cannot provide a proof of convergence of the proposed scheme. However, the following feature of the design deserves to be highlighted.

The design resembles a high-gain observer, but actually involves different mechanisms. In particular, the gain $k$ does not need to be high to ensure convergence ${ }^{5}$. This point is highlighted by the simulations depicted on Figure 5 where the observer was tested on experimental data. The relatively low value of the gain $k$ allows the observer to filter the noisy topside pressure measurement, while providing an accurate estimation of the unmeasured bottom pressure (among other state variables).

The main challenge for the design of observers is now to gain insight into their mechanisms by studying their stability. Possible directions include the use of Lyapunov functions Aamo et al. [2005], possibly non-smooth (see e.g. Clarke [2001]) because of the switching nature of the dynamics. Another option would be to exploit the fact that slugging corresponds to a limit cycle, to extend results on linear oscillators (see, e.g., Praly et al. [2006]).

The observers provide estimates of various state variables that can be used in feedback loops to stabilize slugging systems. In the next section, we review various control strategies which

\footnotetext{
5 Actually, it can be shown that one of the eigenvalues of the linearized error equations of the observer around a trajectory goes to 0 when the gain $k$ goes to infinity. Thus, a large gain $k$ is not desirable.
} 

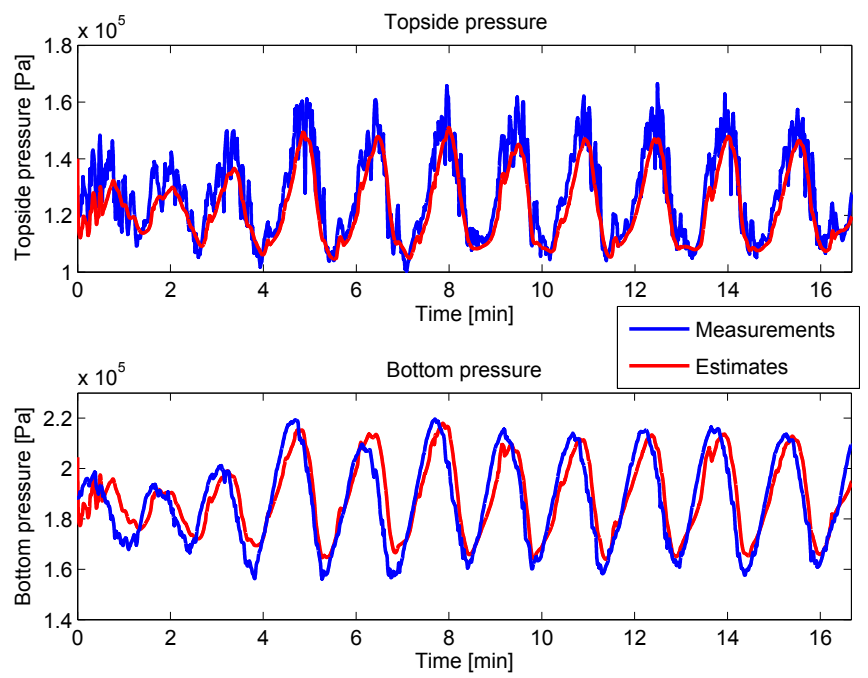

Fig. 5. Simulations of the observer on experimental data, corresponding to slugging oscillations of a 100 meter-long multiphase flow loop. The topside pressure measurement is used in the observer algorithm described by system (9), whereas the bottom pressure measurement is compared to its estimate to evaluate the performances of the observer. Previously, the model has been calibrated using the method described in Di Meglio et al. [2010b] using the same experimental data plotted here.

may rely on such estimate or directly on available measurements.

\section{CONTROL STRATEGIES}

In this section, we describe the state-of-the-art of control strategies for slugging. We consider separately the solutions not relying on any model, i.e. Proportional-Integrator (PI) controllers (possibly cascaded), and model-based control laws.

\subsection{Non model-based strategies}

The first successful stabilization experiments of real-scale slugging wells reported in Courbot [1996] used a PI controller to control the 'Riser Base Pressure' ${ }^{6}$. Since then, numerous implementation of this control strategy have revealed successful Aamo et al. [2005], Dalsmo et al. [2002], Godhavn et al. [2005], Havre et al. [2000], Sinègre [2006], Storkaas [2005]. Tuning directives for this controller, derived from a simplified linear model of the slugging oscillations, can be found in Godhavn et al. [2005].

The PI controller on the bottom pressure exhibits, however, two major shortcomings. First, as reported in Sivertsen et al. [2010] and Di Meglio [2011], it reveals sensitive to the moment where the controller is triggered. More precisely, the controller must be turned on when the bottom pressure is in the increasing phase of its oscillations to ensure its efficiency. Besides, it requires frequent re-tuning due to relatively poor robustness to changes in operating conditions Di Meglio [2011].

In Hedne and Linga [1990], an alternative control variable is considered: the difference between the bottom and topside pressures. This control variable is strongly related to the total mass

\footnotetext{
6 Equivalently, we may refer to this variable as bottom hole, downhole or bottom pressure
}

of liquid in the system (see, e.g., Di Meglio [2011]), which, according to various contributions, plays a critical role in the stability properties of slugging systems Di Meglio et al. [2010a], Imsland [2002], Siahaan et al. [2005]. In Di Meglio [2011], the choice of this control variable is shown to outperform the PI controller on the bottom pressure by allowing stabilization at operating points corresponding to higher levels of production, and without any restriction regarding the triggering time.

Both variables mentioned above require the availability of a bottom pressure sensor. In Sivertsen et al. [2010], non modelbased solutions using topside information only are proposed, under the form of cascaded PI controllers. Various combinations of sensors are considered among the traditionnaly available measurements, namely volumetric flow rate, density, mass flow rate and pressure. The best results are obtained using a normalized volumetric flow rate measurement as the output of the inner loop of the cascade, and the outlet valve opening measurement in the outer loop.

\subsection{Model-based strategies}

In several contributions, models are used solely to provide an estimate of the bottom pressure, which is then stabilized by a PI controller. Such control schemes are described in Aamo et al. [2005], Eikrem et al. [2004], Sinègre [2006].

Other contributions take advantage of the availability of fullstate information provided by the observers to design more advanced control laws, taking into account the nonlinearity of the models. Thus, in Siahaan et al. [2005] and Di Meglio et al. [2010a], control laws are designed to linearize the dynamics of a state variable. In Siahaan et al. [2005], several variables of the Storkaas model are considered, among which the bottom pressure, the height of liquid at the low-point and the mass of liquid in the riser. The latter exhibits the best performances in simulation.

Similarly, in Di Meglio et al. [2010a], Di Meglio [2011], a control law linearizing the dynamics of the mass of liquid in the riser is considered. Presented experiments emphasize the potential of this model-based control law, which performs better than all considered PI strategies.

\section{CONCLUSIONS AND OPEN QUESTIONS}

Given the vast array of explored and unexplored possible solutions, and the diversity of industrial setups, picking one strategy as the best one reveals yet difficult.

The confidence of field practitioners in the easily implementable PI controller makes it remain the favored solution. However, comparison of various output variables, especially the pressure drop over the riser, the inlet pressure of a flowline (see, e.g., Storkaas [2005]), or mass of volumetric flow rates should be performed.

Besides, when no bottom pressure is available, it has been proved in Sivertsen et al. [2010] that no estimate of upstream information is needed to stabilize slugging systems. Experiments comparing cascaded PI controllers on topside measurements with observer-based approaches should be performed to evaluate the benefits of estimating full-state information. Also, a combined approach involving PI controllers cascaded with observer-based feedback laws could be considered. 


\section{REFERENCES}

O.-M. Aamo, G.O. Eikrem, H.B. Siahaan, and B.A. Foss. Observer design for multiphase flow in vertical pipes with gas-lift theory and experiments. Journal of Process Control, 15:247-257, 2005.

S.P.C. Belfroid, R.J. van Der Linden, G.J.N. Alberts, and R. Aasheim R., Schulkes. Severe slugging in oil-well simulations using a drift flux code. pages 357-371, 2010.

K. Bendiksen, D. Maines, R. Moe, and S. Nuland. The dynamic two-fluid model OLGA: Theory and application. SPE production engineering, 6(2):171-180, 1991.

E. F. Blick, P.N. Enga, and P.-C. Lin. Stability analysis of flowing oil wells and gas-lift wells. SPE Production Engineering, 3, No 4:508-514, 1986.

H. H. J. Bloemen, S. P. C. Belfroid, Sturm W. L., and F. J. P. C. M. G. Verhelst. Soft sensing for gas lift wells. SPE Paper, 2006.

C. E. Brennen. Fundamentals of multiphase flow. Cambridge Univ Press, 2005.

F. Clarke. Nonsmooth analysis in control theory: a survey. European Journal of Control, 7(2-3):145-159, 2001. ISSN 0947-3580.

A. Courbot. Prevention of severe slugging in the dunbar 16' multiphase pipeline. Offshore Technology Conference, 1996.

C. M. Da Silva and O. J. Nydal. Dynamic multiphase flow models for control. BHRG 7th North American Conference on Multiphase Technology, Banff, Canada, 2010.

M. Dalsmo, E. Halvorsen, and O. Slupphaug. Active feedback control of unstable wells at the brage field. SPE Annual Technical Conference, 2002.

F. Di Meglio. Dynamics and control of slugging in oil production. PhD thesis, MINES ParisTech, 2011.

F. Di Meglio, G.-O. Kaasa, and N. Petit. A first principle model for multiphase slugging flow in vertical risers. Conference on Decision and Control, 2009.

F. Di Meglio, G.-O. Kaasa, N. Petit, and V. Alstad. Modelbased control of slugging flow: an experimental case study. 2010 American Control Conference, 2010a.

F. Di Meglio, G.-O. Kaasa, N. Petit, and V. Alstad. Reproducing slugging oscillations of a real oil well. 49th IEEE Conference on Decision and Control, 2010b.

F. Di Meglio, G.-O. Kaasa, N. Petit, and V. Alstad. Slugging in multiphase flow as a mixed initial-boundary value problem for a quasilinear hyperbolic system. 2011 American Control Conference, 2011.

F. Di Meglio, R. Vazquez, M. Krstic, and N. Petit. Backstepping stabilization of an underactuated $3 \times 3$ linear hyperbolic system of fluid flow transport equations. 2012 American Control Conference, Montreal, Canada (to appear), 2012.

E. Duret. Dynamique et contrôle des écoulements polyphasiques. $\mathrm{PhD}$ thesis, Ecole des Mines de Paris, 2005.

G. O. Eikrem, B. Foss, L. Imsland, B. Hu, and M. Golan. Stabilization of gas lifted wells. Proceedings of the 15th IFAC World Congress, 15, Part 1, 2002.

G. O. Eikrem, L. Imsland, and B. Foss. Stabilization of gas-lifted wells based on state estimation. International Symposium on Advanced Control of Chemical Processes, 2004.

J.-M. Godhavn, M. P. Fard, and P. H. Fuchs. New slug control strategies, tuning rules and experimental results. Journal of Process Control, 15:547-557, 2005.

K. Havre, K.O. Stornes, and H. Stray. Taming slug flow in pipelines. ABB review, 4:55-63, 2000.
P. E. Hedne and H. Linga. Suppression of terrain slugging with automatic and manual riser choking. Presented at the ASME Winter Annual Meeting, Dallas, Texas, 1990.

V. Henriot, E. Duret, E. Heintz, and A. Courbot. Multiphase production control: Application to slug flow. Oil $\mathcal{E}$ Gas Science and Technology - Rev IFP, 57, No. 1:87-98, 2002.

B. Hu. Characterizing gas-lift instablilities. PhD thesis, Department of Petroleum Engineering and Applied Geophysics, NTNU, 2004.

L. S. Imsland. Output Feedback and Stabilization and Control of Positive Systems. PhD thesis, Norwegian University of Science and Technology, Department of Engineering Cybernetics, 2002.

E. Jahanshahi and S. Skogestad. Simplified dynamical models for control of severe slugging in multiphase risers. Preprints of the 18th IFAC World Congress, Milano, Italy, 2011.

B. Jansen, M. Daslmo, L. Nøkleberg, K. Havre, V. Kristiansen, and P. Lemetayer. Automatic control of unstable gas lifted wells. SPE annual technical conference, 1999.

G.-O. Kaasa, V. Alstad, J. Zhou, and O. M. Aamo. Nonlinear model-based control of unstable wells. Modeling, Identification and Control, 28(3):68-77, 2007.

T. Kailath, A. H. Sayed, and B. Hassibi. Linear Estimation. Prentice-Hall, Inc., Upper Saddle River, NJ, 2000.

M. Krstic and A. Smyshlyaev. Backstepping boundary control for first-order hyperbolic pdes and application to systems with actuator and sensor delays. Systems $\mathcal{E}$ Control Letters, 57(9):750 - 758, 2008a. ISSN 0167-6911.

M. Krstic and A. Smyshlyaev. Boundary Control of PDEs. SIAM Advances in Design and Control, 2008b.

L. Praly, A. Isidori, and L. Marconi. A new observer for an unknown harmonic oscillator. Proceedings of the 17th International Symposium on Mathematical Theory of Networks and Systems, Kyoto, Japan, July 24-28 2006.

Z. Schmidt, J.P. Brill, and H.D. Beggs. Choking can eliminate severe pipeline slugging. Oil $\mathcal{F}$ Gas Journal, 12:230-238, 1979.

F. Scibilia, M. Hovd, and R. R. Bitmead. Stabilization of gaslift oil wells using topside measurements. Proceedings of the 17th IFAC World Congress, pages 13907-13912, 2008.

H. B. Siahaan, O. M. Aamo, and Foss B. A. Suppressing riserbased slugging in multiphase flow by state feedback. Proceedings of the 44th Conference on Decision and Control, pages 12-15, 2005.

L. Sinègre. Dynamic study of unstable phenomena stepping in gas-lift activated systems. $\mathrm{PhD}$ thesis, Ecole des Mines de Paris, 2006.

H. Sivertsen, E. Storkaas, and S. Skogestad. Small-scale experiments on stabilizing riser slug flow. Chemical Engineering Research E Design, 88(2A):213-228, 2010.

E. Storkaas. Control solutions to avoid slug flow in pipelineriser systems. PhD thesis, Norwegian University of Science and Technology, 2005.

E. Storkaas and S. Skogestad. Stabilization of severe slugging based on a low-dimensional nonlinear model. AIChE Journal, 2002.

R. Vazquez, M. Krstic, and J.-M. Coron. Backstepping boundary stabilization and state estimation of a $2 \times 2$ linear hyperbolic system. in Proceedings of the 50th Conference on Decision and Control, Orlando, 2011.

E. Zakarian. Analysis of two-phase flow instablilities in piperiser systems. Proceedings of Pressure Vessels and Piping Conference, 2000. 